\title{
Die passive Übertragung der Soforttyp-Allergie im Selbstversuch durch Carl Prausnitz und Heinz Küstner - ein Meilenstein in der Allergieforschung
}

\author{
The Passive Transfer of Immediate Type Allergy in the Experiment on Themselves by Carl Prausnitz and \\ Heinz Küstner - A Milestone in the Research on Allergies
}

Autor

Institut

\section{H.-D. Göring}

Tumorzentrum Anhalt am Städtischen Klinikum Dessau e. V. (Vorsitzender: Prof. Dr. med. H.-D. Göring)

\section{Bibliografie}

DOI $10.1055 / \mathrm{s}-2007-966226$

Akt Dermatol 2007; 33:

87-91 ๑ Georg Thieme

Verlag KG Stuttgart · New York ISSN 0340-2541

Korrespondenzadresse

Prof. Dr. med. Hans-Dieter Göring

Tumorzentrum Anhalt am Städtischen Klinikum

Dessau e. V.

Auenweg 38, 06847 Dessau

tzd@klinikum-dessau.de

\section{Zusammenfassung \\ $\nabla$}

1921 veröffentlichten Carl Prausnitz und sein Assistent Heinz Küstner die Ergebnisse ihrer Selbstversuche zur passiven Übertragung der Soforttyp-Allergie. Die Reproduzierbarkeit dieses Prausnitz-Küstner-Versuches wurde in der Folgezeit von anderen Untersuchern bestätigt. Der Prausnitz-Küstner-Versuch gilt aus heutiger Sicht als Startpunkt des Studiums der Natur hautsensibilisierender Antikörper und ihrer Nachweismethoden. Zunächst von Coca als Rea-

1921 teilten Carl Prausnitz und Heinz Küstner in einem Artikel die Ergebnisse ihrer Selbstversuche zur Übertragung der Soforttyp-Allergie mit (๑ Abb. 1) [1]. Der Prausnitz-Küstner-Versuch (auch -Reaktion, -Test oder -Phänomen) gilt seither als Meilenstein in der Allergieforschung und als ein Basisversuch der Klinischen Immunologie. Die Veröffentlichung im Zentralblatt für Bakteriologie machte die Autoren international bekannt [2]. Die Geschichte des Prausnitz-KüstnerVersuches und die Biografien seiner Inauguratoren reflektieren einen wichtigen Abschnitt der Medizin- und Zeitgeschichte und verdienen in unserem Gedächtnis bewahrt zu werden.

Carl Wilhelm Prausnitz wurde am 12. Oktober 1871 als Sohn eines deutschen Arztes und einer englischen Mutter in Hamburg geboren ( Abb. 2). Die Familie der Mutter lebte in Bonchurch auf der Isle of Wight. Der Ort war dadurch bekannt, dass Charles Dickens hier 1849 ein Haus erworben hatte und die ersten Kapitel seines autobiografischen Romanes „David Copperfield“ verfasst hatte [3]. Carl Prausnitz verlebte hier als Kind bei seinen Verwandten oft seine Ferien und wuchs bilingual auf [3]. Nach Schulbesuchen in Hamburg, Weymouth und Darmstadt bestand er

${ }^{*}$ Herrn Prof. Dr. med. E. G. Jung zum 75. Geburtstag. gine bezeichnet und als ein Charakteristikum der Atopie erkannt, konnten die Antikörper 1967 von K. und T. Ishizaka als IgE identifiziert werden. Der Prausnitz-Küstner-Versuch ist ein Basisversuch der Klinischen Immunologie und ein Meilenstein in der Allergieforschung. Er wurde bis zur Entdeckung des IgE in Praxis und Forschung eingesetzt. Wegen der deutlichen Einschränkungen seiner Anwendung infolge einer möglichen Übertragung von Infektionskrankheiten und des technisch einfacheren IgE-Nachweises besitzt er heute keine praktische Bedeutung mehr.

1896 das Abitur und studierte anschließend Medizin in Leipzig, Kiel und Breslau, wo er 1901 das Staatsexamen bestand und 1902 mit einer Arbeit über Choleravibrionen mit „summa cum lauda“ zum Dr. med. promovierte [2,3]. Von 1902 bis 1905 arbeitete Prausnitz am Staatlichen Hygieneinstitut Hamburg unter Professor William Dunbar. Neben der medizinischen Mikrobiologie galt Dunbars Interesse dem Heufieber, wie damals der Heuschnupfen überwiegend bezeichnet wurde. Dunbar war der Ansicht, dass der Heuschnupfen auf die Wirkung eines Toxins in Pollen zurückzuführen sei [3]. Dunbar und Prausnitz litten selbst an einer Pollenüberempfindlichkeit $[2,3]$. Sie beobachteten im Selbstversuch, dass sich durch lokale Applikation von Graspollen in der Nasenschleimhaut und den Augenbindehäuten eine Heuschnupfensymptomatik wiederholt auslösen ließ. Wenn sich Prausnitz eine Pollenlösung subkutan injizierte, entwickelte er unmittelbar darauf schwere Asthmaanfälle, generalisierte Urtikaria und Angioödeme [3,4]. Als Dunbar darauf bestand, dieses Experiment bei ihm zu wiederholen, wäre ihm das wegen des dadurch provozierten Asthmaanfalls und Kreislaufkollapses fast zum Verhängnis geworden, zumal Adrenalin und andere Notfallmedikamente nicht zur Verfügung standen [3,4]. „Ich wäre fast zum 


\section{Studien über die Ueberempfindlichkeit.}

Nachdruck verboten.

[Aus dem Hygienischen Institut der Universität Breslau (Direktor: Geh. Med.-Rat Prof. Dr. R. P f e iffer).]

\section{Von Carl Prausnitz und Heinz Kïstner.}

Von den Idiosynkrasien gegen Nahrungsmittel ist die Ueberempfindlichkeit gegen Fischeiweiß bisher wenig studiert worden. Da der eine von uns (K.) mit dieser Anomalie behaftet ist, benutzten wir die Gelegenheit zur Ausführung von Untersuchungen über den Mechanismus der Reaktion und die dabei vorliegenden serologischen Verhältnisse. Dabei
Abb. 1 Veröffentlichung des Prausnitz-KüstnerVersuches 1921 [1].

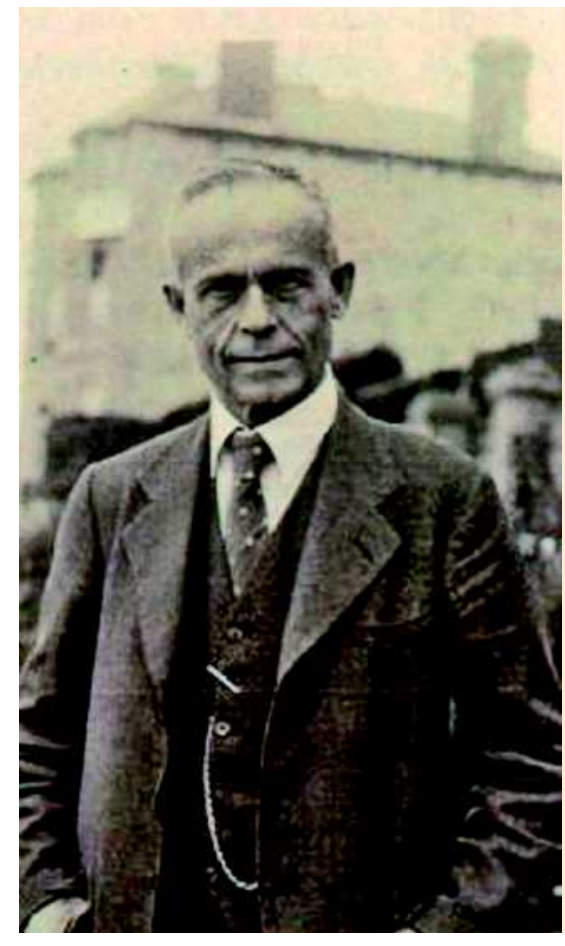

Abb. 2 Prof. Dr. med. Carl Prausnitz-Giles.

Mörder meines Chefs geworden“, resümierte Prausnitz 50 Jahre später [4]. Dunbar und Prausnitz gelang es, Pferde, Kaninchen und Ziegen aktiv gegen Pollen zu sensibilisieren. Die so gewonnenen Immunseren, die sie „Pollentine“ nannten, waren bei lokaler Anwendung an den Augenbindehäuten und der Nasenschleimhaut in der Lage, die Pollenwirkung zu neutralisieren. Damit hatten sie Dunbars anfängliche Ansicht einer reinen Toxinwirkung der Pollen zugunsten eines immunologischen Mechanismus widerlegt. Dunbar und Prausnitz gaben die Behandlung des Heuschnupfens mit Pollentinen auf, als sich einige Patienten gegen Pferdeseren sensibilisiert hatten $[3,5]$.

Neben Dunbar und Prausnitz beschäftigten sich fast zeitgleich eine Reihe anderer Wissenschaftler an mehreren Orten in Europa mit dem Phänomen der Überempfindlichkeit [2-4,6], so dass hier der Begriff des „Elementargedankens“, wie ihn der Düsseldorfer Medizinhistoriker Hans Schadewaldt formulierte, Anwendung finden kann. So beschrieben u.a. 1902 Richet und Portier die Anaphylaxie genannte tödliche Reaktion eines Hundes auf wiederholte Injektionen eines Actinotoxins aus Seeanemonen. Richet erhielt 1913 für die Ergebnisse der Anaphylaxiestudien den Nobelpreis [3]. 1902 beschrieb der Wiener Pädiater Clemens von Pirquet zusammen mit Bela Schick das Krankheitsbild der Serumkrankheit nach Gabe von in Impfstoffen enthaltenen
Fremdeiweißen. Er vermutete einen zugrunde liegenden immunologischen Mechanismus und nannte das Phänomen $1906 \mathrm{Al}-$ lergie $[3,4,7]$. 1906 wies Alfred Wolff-Eisner darauf hin, dass es sich bei Heuschnupfen um eine Form der Überempfindlichkeit oder Anaphylaxie der Nase handele, 1910 erweiterte Samuel Meltzer diesen Hinweis auf das Asthma bronchiale. 1911 berichtete Noon erstmals über Untersuchungen zur Desensibilisierung von 20 Heuschnupfenpatienten in einer offenen Studie [3]. Die Aufzählung von Untersuchungen zum Phänomen der Überempfindlichkeit aus dieser Zeit ließe sich beliebig fortsetzen [4].

Von 1905 bis 1908 arbeitete Prausnitz als Demonstrator für Bakteriologie am Royal Institute of Public Health in London. Neben seiner Arbeit als Bakteriologe übersetzte er Vortragsmanuskripte und Publikationen zur Veröffentlichung in der Institutszeitschrift. Hierdurch lernte er auch bekannte Wissenschaftler wie Ilja Metschnikoff kennen und schloss mit einigen von ihnen, wie z.B. Paul Ehrlich und Richard Pfeiffer, lebenslange Freundschaften [3]. In dieser Zeit erwarb Prausnitz die britische Approbation als Arzt. Nach einer 2-jährigen Tätigkeit als Bakteriologe am Metropolitan Asylums Board in London folgte Prausnitz 1910 einer Einladung von Professor Richard Pfeiffer zu gemeinsamer Arbeit an das Hygieneinstitut der Universität Breslau, dessen Direktor Pfeiffer war. Pfeiffer genoss durch die Entdeckung des Hämophilus influenzae und der Bakteriolysine sowie die Einführung der Typhusschutzimpfung international ein hohes Ansehen. 1912 habilitierte sich Prausnitz und folgte 1914 nach Ausbruch des 1 . Weltkrieges seinem Chef, der als beratender Hygieniker eines kaiserlichen Armeekorps eingezogen worden war. Es wird berichtet, dass Prausnitz und Küstner auf der Suche nach Tetanusserum im Pasteur-Institut von Lille auf Leon Calmette und Camille Guerin trafen, die unter Spionageverdacht festgenommen worden waren. Ihnen wurde vorgeworfen, eine Taube zur Übermittlung geheimer Informationen benutzt zu haben, was beide bestritten. Prausnitz soll aufgrund seiner Französischkenntnisse in den Laborprotokollen des Instituts den Beweis gefunden haben, dass die inkrimierte Taube für die Entwicklung von BCG gedient hatte und nicht als Kurier für geheime Botschaften. Dieses Rechercheergebnis habe zur sofortigen Freilassung der beiden französischen Kollegen geführt [2,3].

Nach Kriegsende kehrte Prausnitz wieder an das Hygieneinstitut in Breslau zurück. Hier war vom 15. 4. bis 15.10.1920 der 23-jährige Dr. med. Joachim Heinrich („Heinz“) Küstner als Volontärassistent bei Prof. Pfeiffer angestellt. Heinz Küstner war am 16. Januar 1897 in Breslau geboren worden ( Abb.3). Er war der Sohn des Gynäkologen Prof. Otto Küstner („Küstnersches Lösungszeichen“ der Plazenta), der 30 Jahre als Direktor der Frauenklinik und zeitweise als Rektor der Universität Breslau wirkte. Nach dem Abitur 1914 in Breslau studierte Heinz Küstner in sei- 


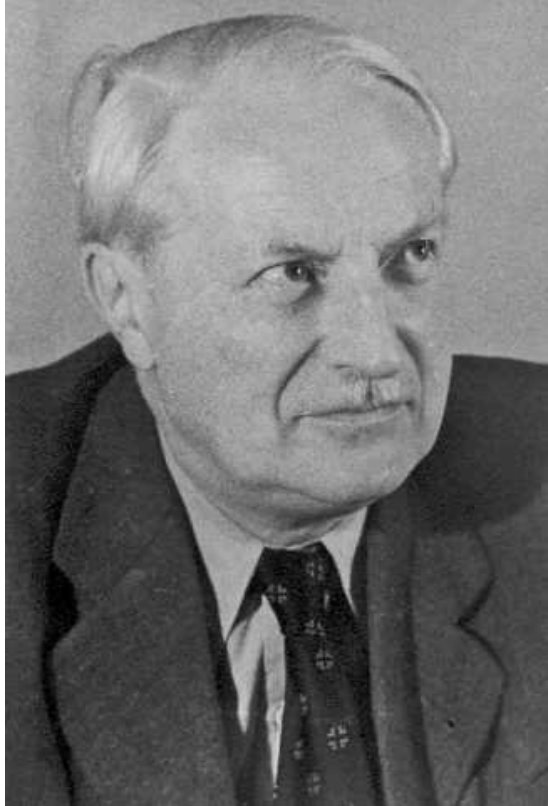

Abb. 3 Prof. Dr. med. Heinz Küstner.

ner Geburtsstadt Medizin und wurde bereits nach 6 Semestern als Unterarzt zum Militärdienst eingezogen. Nach 8 Semestern bestand er 1919 das medizinische Staatsexamen mit „sehr gut“ und promovierte 1921 mit einer Dissertation über die Placenta praevia zum Dr. med. [9]. Von 1919 bis 1923 war er Assistent an der Klinik seines Vaters, 1920 unterbrochen durch die halbjährige Volontärarztzeit im Hygieneinstitut.

In diese Zeit fallen die von Prausnitz initiierten und geplanten gemeinsamen Untersuchungen zur passiven Übertragung der Soforttyp-Allergie. Küstner erschien Prausnitz wegen seiner hochgradigen Fischallergie als Spender des zu untersuchenden Serums geeignet. Seit seinem 6. Lebensjahr reagierte Küstner auf den Genuss selbst kleinster Mengen von gekochtem Fisch mit generalisierter Urtikaria, Rhino-Konjunktivitis und Asthmaanfällen [9]. Im Intrakutantest betrug die wirksame Grenzkonzentration für einen positiven Ausfall bei Küstner 0,01 mg Fischextrakt [1].

Frühere Versuche von Prausnitz, die Allergie vom Soforttyp mit Serum eines Allergikers durch intraperitoneale Applikation passiv auf Meerschweinchen zu übertragen und eine Hautreaktion durch ausschließlich intrakutane Injektion des Allergens auszulösen, waren negativ verlaufen [1]. Eine Anwendung dieser Versuchsanordnung beim Meerschweinchen auf den Menschen kam wegen der großen Menge des zu übertragenden Spenderserums und der Gefahr eines anaphylaktischen Schocks beim Empfänger aus ethischen Gründen nicht in Betracht. Prausnitz kannte aus der Literatur den Fall einer von Ramirez 1919 beschriebenen ungewollten passageren Übertragung einer Pferdehaarallergie durch eine Bluttransfusion [4].

Prausnitz hatte bei seinen gemeinsam mit Küstner durchgeführten Experimenten den festen Vorsatz, den Empfänger durch passive Übertragung der Soforttyp-Allergie nicht dauerhaft und systemisch zu sensibilisieren [4,7]. Er wählte daher die Versuchsbedingungen so, dass die iatrogene allergische Reaktion auf die Haut begrenzt blieb und nach kürzerer Zeit erlosch. Er selbst ließ sich in seinen Unterarm das Serum des Fischallergikers Küstner intrakutan injizieren und 24 Stunden später in gleicher Lokalisation den Extrakt aus gekochtem Fisch [1]. Es kam bei ihm und später bei 2 weiteren Versuchspersonen zu positiven Hautreaktionen. Häufige Wiederholung des Versuches im glei- chen Testareal führte zu einer wochenlang anhaltenden Abschwächung der Reaktionen im Testareal [1]. Es gelang nicht, mit der oben beschriebenen Versuchsanordnung die Empfindlichkeit gegen Pollen, Tuberkulin und Pferdeseren zu übertragen. Bei der Prüfung der passiven Übertragung der Pollenüberempfindlichkeit wurde Serum des Pollenallergikers Prausnitz in den Unterarm von Küstner injiziert und 24 Stunden später ein Pollenextrakt. Es wurde spekuliert, dass der Grad der Überempfindlichkeit gegen Pollen ebenso wie gegen Tuberkulin und Pferdeserum nicht hoch genug gewesen sein könnte [1]. Die tatsächliche Erklärung für den negativen Reaktionsausfall lieferte später Küstner, der 1963 seinem Kollegen Prausnitz anlässlich dessen Besuches in Leipzig gestand, dass er das Serum von Prausnitz aus Furcht vor der Übertragung einer eventuellen Erkrankung erhitzt habe, bevor er es sich als Testperson intrakutan injizieren ließ [8]. Dass durch den Erhitzungsvorgang auch die Substanz, die beim passiven Transfer die Überempfindlichkeit übertrug, zerstört werden könnte, war Küstner zum Zeitpunkt des Versuches 1920 offenbar nicht bewusst. Rückblickend erinnerte sich Prausnitz daran, dass er 1920 den Eindruck hatte, Küstner interessiere sich nur wenig für Allergologie und dass es einer gewissen Überredungskunst bedurft hatte, ihn zu den Experimenten zu bewegen $[2,8]$.

Mit dem Prausnitz-Küstner-Versuch gelang 1920 die passive Übertragung der Fischüberempfindlichkeit auf eine nicht fischempfindliche Person. Die Reaktion war streng spezifisch. Präzipitine, komplementbindende und neutralisierende Stoffe waren im Serum der überempfindlichen Person nicht nachweisbar. Die wirksame Substanz war in Alkohol und Äther sowohl in der Kälte wie in der Wärme unlöslich, sie war nicht dialysierbar und wurde durch Säuren rasch unwirksam, durch Alkali, Pepsin und Trypsin aber nicht abgeschwächt [1]. Der Prausnitz-KüstnerVersuch wird heute als Startpunkt des Studiums der Natur hautsensibilisierender Antikörper bei Allergien und ihrer den Patienten schonenden Nachweismethoden betrachtet [6].

In der Zeit nach der Veröffentlichung des Prausnitz-Küstner-Versuchs griffen andere Autoren die Methode auf. De Besche gelang damit 1923 der passive Transfer einer Allergie gegen Pferdeepithelien und Pollen [10] und Freeman 1924 ebenfalls bei einer Allergie gegen Pollen [11]. In eigenen Versuchsserien mit dem Prausnitz-Küstner-Test haben Coca und Grove 1925 festgestellt, dass in $11 \%$ ihrer Untersuchungen der passive Transfer einer Pollenallergie nicht gelang. Sie beschrieben auch, dass die hautsensibilisierende Eigenschaft des Spenderserums durch Erhitzen aufgehoben wird [12]. Für die Stoffe, die durch den positiven Intrakutantest und die positive Übertragung einer Soforttyp-Allergie nachgewiesen werden, prägte Coca den Begriff „Reagine“ [6]. Coca und Grove konnten die Reagine klar von anaphylaktischen Antikörpern differenzieren und detailliert charakterisieren, wobei sie die von Prausnitz und Küstner beschriebenen Eigenschaften bestätigten [12]. Atopische Reagine wurden nach Untersuchungen von Levine und Coca 1925 offenbar bei einer entsprechenden hereditären Disposition selbst gebildet und waren in vivo langlebig. Es schien, dass sie eine große Rolle bei Heuschnupfen, Asthma und atopischem Ekzem spielen [13]. Diese Allergieform wurde von Coca als „Atopie“ bezeichnet [4]. Parallel durchgeführte Prausnitz-Küstner-Versuche erleichterten die Abgrenzung von allergischen zu irritativen und anderen falschpositiven Intrakutantests [13]. In einigen Fällen wurde eine Überempfindlichkeit auf Quecksilber, Wismut, Salvarsan, Insulin und Leberextrakt passager und nicht regelmäßig im PrausnitzKüstner-Test nachgewiesen. Ihre klinische Relevanz bei fehlen- 
der Atopie war nicht eindeutig [6]. Reagine wurden mit dem Prausnitz-Küstner-Versuch auch im Nasensekret und Colostrum nachgewiesen, aber nicht im Nabelschnurblut und in der Zerebrospinalflüssigkeit [6]. Technische Modifikationen des Prausnitz-Küstner-Versuchs betrafen den autopassiven Transfer der Reagine in die patienteneigene Haut mit Allergeninjektionen in entferntere Hautareale sowie die Serumgewinnung nach peroraler Gabe von Nahrungsmitteln unter der Annahme einer Entstehung der eigentlichen Allergene durch die Verdauung [14]. Praktische Anwendung fand der Prausnitz-Küstner-Versuch bei Patienten mit ausgedehntem Hautbefall, aus Angst vor Intrakutantests und mit eingesandten Serumproben $[6,14]$. Für wissenschaftliche Zwecke wurden Verdünnungen von Seren und Allergenen vorgenommen. Ein Resultat solcher Untersuchungen waren indirekte Hinweise auf die Wirkung blockierender Antikörper im Verlauf von Injektionsbehandlungen mit Ragweed-Allergenen bei entsprechend sensibilisierten Patienten [15]. Bis zur Entdeckung des IgE 1967 blieb der Prausnitz-Küstner-Versuch 45 Jahre in der Diagnostik von allergischen Krankheitsbildern und der Allergieforschung in Gebrauch - allerdings mit deutlicher Einschränkung, v.a. wegen der Gefahr der Übertragung von Lues, Hepatitis und anderen Infektionskrankheiten [6]. Küstner hat 1924 in einem kurzen Artikel darüber berichtet, dass seine Fischallergie seit den Versuchen mit Prausnitz 1920 deutlich abgenommen hatte. Er könne jetzt Fisch essen und der Intrakutantest mit Fischextrakt sei wesentlich schwächer als früher. Küstner führte das auf eine Umstimmung durch die häufigen Intrakutantestungen zurück [16]. Zum Zeitpunkt dieser Veröffentlichung war er bereits Assistent an der Universitäts-Frauenklinik Halle bei Prof. Sellheim, wo er seine Facharztausbildung abschloss und sich 1924 habilitierte. Mit Sellheim wechselte er an die Universitäts-Frauenklinik Leipzig und wurde 1929 zum a. o. Professor ernannt. 1936 übernahm er die Leitung der gynäkologisch-geburtshilflichen Klinik „Bethanien“ in Leipzig. Im 2. Weltkrieg war Küstner Chef eines Lazarettzuges. Er verfasste in dieser Zeit das später bei Studenten beliebte Lehrbuch „Gynäkologie und Geburtshilfe“, das bis 1960 zehn Auflagen erlebte [9]. Nach 1945 folgten für Heinz Küstner Stationen als Landarzt, Leiter eines Flüchtlingslagers sowie eines Typhuskrankenhauses, ehe er sich wieder seinem Fach zuwenden konnte und zum Chefarzt der gynäkologisch-geburtshilflichen Abteilung und Ärztlichen Direktor des Kreiskrankenhauses Weißenfels berufen wurde. 1954 wurde er für seine Verdienste um das Gesundheitswesen als „Verdienter Arzt des Volkes“ geehrt. 1958 zwang ihn eine fortschreitende Erkrankung zur Aufgabe seiner Tätigkeit in Weißenfels. Prof. Küstner verbrachte seine letzten Lebensjahre in Leipzig, wo er zeitweise noch ambulant tätig war und am Zentralblatt für Gynäkologie und Geburtshilfe mitarbeitete. 1963 besuchte ihn Prausnitz in Leipzig. Am 21. 8. 1966 verstarb Heinz Küstner im Alter von 69 Jahren in Leipzig [9].

Prausnitz wurde 1923 als Professor und Stellvertretender Direktor des Hygieneinstituts der Universität Greifswald berufen, 1926 als Direktor des Hygieneinstituts Breslau als Nachfolger seines Lehrers Richard Pfeiffer. Gleichzeitig arbeitete er in der Gesundheitsorganisation des Völkerbundes und knüpfte enge Kontakte zu ausländischen Kollegen. Prausnitz galt als aussichtsreichster Kandidat für den renommierten Lehrstuhl seines Faches an der Berliner Universität [2,3]. Wegen einer Reise in die Sowjetunion wurde er kommunistischer Sympathien verdächtigt. Der heraufziehende Nationalsozialismus stellte für ihn eine Gefahr dar, weil sein Großvater väterlicherseits Jude gewesen war. Als Prausnitz nach einer Auslandsreise kurzzeitig inhaftiert wurde, verließ er 1933 mit seiner Familie Breslau und ging nach Großbritannien. 1934 gab er seinen Lehrstuhl in Breslau auf $[2,3]$. Mit einem Stipendium der Rockefeller-Stiftung arbeitete er am Hygieneinstitut in Manchester an der Aufklärung des sog. Asthmas der Baumwollspinner, der Byssinose [2 -4]. Im Tierversuch konnte er eine allergische Alveolitis infolge der Exposition mit Baumwollstaub nachweisen. Bis dahin hatte die Ansicht gegolten, dass das im Staub enthaltene Histamin für diese Lungenerkrankung verantwortlich sei. Beim Patienten unterschied sich der Intrakutantest mit Baumwollstaub von dem mit Gräserpollen durch den zeitlichen Ablauf $[2,3,17]$. Eine endgültige Abklärung der Byssinose durch Prausnitz scheiterte an der fehlenden finanziellen Unterstützung [2]. Ein in dieser Zeit ergangenes Angebot auf einen Lehrstuhl in Zürich lehnte Prausnitz ab. Er kaufte sich stattdessen in die allgemeinmedizinische Praxis seines Jugendfreundes Dr. Bruce Williams in Ventnor auf der Isle of Wight ein und nahm den Geburtsnamen seiner Mutter, Giles, an. [2,3]. 1939 wurde er britischer Staatsbürger. Carl Prausnitz-Giles bezeichnete später seine Jahre als Allgemeinmediziner als die glücklichsten seines Lebens. Lediglich die Geburtshilfe bereitete ihm gelegentlich Kopfschmerzen. Seine Patienten liebten und verehrten ihn. Trotz seiner anstrengenden Tätigkeit als praktischer Arzt blieb Prausnitz der Allergologie und Klinischen Immunologie verbunden. Er gehörte 1954 zu dem exklusiven Kreis von Gründungsmitgliedern des Collegium Internationale Allergologicum. In Großbritannien wird er als „Vater der Klinischen Immunologie“" verehrt [2]. Obwohl sich Prausnitz 1933 geschworen hatte, nie wieder deutschen Boden zu betreten, folgte er 1958 einer Einladung Karl Hansens zur Überreichung des Aronson-Preises des Robert Koch-Instituts in Berlin und 1960 zur Entgegennahme der Ehrendoktorwüde der Universität Hamburg [2,3]. Am 21. 4.1963 starb Carl Prausnitz-Giles in Ventnor, Isle of Wight. Noch auf seinem Totenbett hatte Prausnitz-Giles einen Brief zur Frage der Beeinflussung der Allergie durch Hypnose diktiert, der nach seinem Tod im British Journal of Medicine erschien [2].

\section{Danksagung \\ $\nabla$}

Für wertvolle Angaben zur Biografie seines Vaters Prof. Dr. med. Heinz Küstner sowie die Überlassung der Fotografie bin ich Herrn Dr. med. Roland Küstner, Erfurt, zu besonderem Dank verpflichtet. Herrn Dr. med. Alan Champion, Ventnor on Isle of Wight, danke ich herzlich für seine Angaben zu Prof. Dr. med. Carl Prausnitz-Giles als Allgemeinmediziner in Ventnor.

\section{Abstract}

The Passive Transfer of Immediate Type Allergy in the Experiment on Themselves by Carl Prausnitz and Heinz Küstner - A Milestone in the Research on Allergies $\nabla$

Carl Prausnitz and his assistant Heinz Küstner published the results of the experiments on themselves concerning the passive transfer of the immediate type allergy in 1921. The replicability of this experiment has subsequently been confirmed by other researchers. From today's perspective the Prausnitz-Küstner Experiment can be seen as the starting point of the research on skin sensitizing antibodies and their detection methods. Initially cal- 
led Reagine by Coca and seen as a feature of atopy, the antibodies have been identified as IgE by K. and T. Ishizaka in 1967. The Prausnitz-Küstner Experiment is a basic experiment in the clinical immunology and a milestone in the research on allergies. It was used in both research and practice until the discovery of IgE. As a result of the notable limitations in use due to possible transmissions of infectious diseases and the technically simpler IgE detection, it does not have any practical significance today.

\section{Literatur}

1 Prausnitz C, Küstner H. Studien über die Ueberempfindlichkeit. Centralbl Bakt I. Abt Originale 1921; 86: 160-169

2 Frankland AW. Carl Prausnitz: A personal memoir. J Allergy Clin Immunol 2004; 114: 700 - 704

3 Hide DW. Carl Prausnitz - Father of Clinical Allergy. Southampton Med J 1992; 8: 48-51

4 Prausnitz C. Einst und jetzt: Die passive Übertragung der Allergie. Münch med Wochenschr 1958; 100: 1313-1316

5 Dunbar $W$. The present state of our knowledge of hay-fever. J Hyg 1913; 13: 105 - 118

6 Cohen SG, Zelaya-Quesada M. Prausnitz and Küstner phenomenon: the P-K reaction. J Allegy Clin Immunol 2004; 114: 705 - 710
7 Piquet $C v$. Klinische Studien über Vakzination und vakzinale Allergie. Münch med Wochenschr 1906; 53: 1457 - 1460

8 Frankland AW. Allergy: immunity gone wrong. Proc Royal Soc Med 1973; 66: $365-368$

9 Küstner R. Persönliche Mitteilung

10 De Besche A. Studies on the reactions of asthmatics on a passive transfer of hypersensitivity. Am J Med Sci 1923; 166: 265 - 275

11 Freeman J. Discussion on paroxysmal rhinorrhoea. Proc Royal Soc Med Sec Laryngol 1924; 18: 29-32

12 Coca AF, Grove EE. Studies in hypersensitiveness: XIII. A study of the atopie reagine. J Immunol 1925; 10: 445 - 463

13 Levine P, Coca $A F$. Studies in hypersensitiveness: XX: A quantitative study of the interaction of atopic reagin and atopen. J Immunol 1926; 11: $411-433$

14 Walzer M. The indirect method of testing (passive transfer). In: Cooke RA (ed). Allergy in theory and practice. Philadelphia: Sounders, 1947: 507-510

15 Cooke RA, Barnard JH, Hebald S, Stull A. Serological evidence of immunity with coexistent sensitization in a type of human allergy, hay fever. J exp Med 1935; 69: 723 - 750

16 Küstner H. Studien über die Ueberempfindlichkeit. Centralbl Bakt I. Abt Originale 1924; 92: $428-429$

17 Prausnitz C. Investigations on respiratory dust diseases in operatives in the cotton industry. Med Res Council 1936; Spec Rep Series no 212:

\section{Buchbesprechung}

Paul Klee und seine Krankheit. Vom Schicksal geschlagen, vom Leiden gezeichnet - und dennoch!

H. Suter

Bern: Stämpfli, 2006. 272 S., 190 Abb. Geb., 37,00€, CHF 55,00

ISBN 3-7272-1106-7

ISBN 978-3-7272-1106-5

Medizin gehört wenigstens zum Teil in die Kulturgeschichte und Personen der Kulturgeschichte und der Kunst im Besonderen gehören mit ihren Krankheiten in die Medizin. So ist Kunst und Kultur über die tragenden Personen mit der Medizin eng verbunden. Dies wird am Beispiel von Paul Klee (1879-1940) dargestellt, einem der hervorragenden und vielseitigsten Maler des 20. Jahrhunderts. Dieser litt die letzten 5 Jahre seines Lebens unter einer progressiv verlaufenden diffusen Sklerodermie, die damals zwar beschrieben, nicht aber effektiv behandelt werden konnte. Das gigantische Lebenswerk von Paul Klee erfuhr international erst langsam die große Beachtung, die diesem unzweifelhaft zusteht. Ausstellungen und Bücher, beide oft nach inhaltlichen Kriterien oder nach den Ausdrucksformen zusammengestellt, bereichern die Sicht auf den Künstler, dem nun seit 2005 das von der Familie Klee und von Maurice und Martha Müller-Lüthi gestiftete Zentrum Paul Klee als „Monument im Fruchtland“ bei Bern die künstlerische Heimstatt bietet. Sammlung, Dokumentation, Forschung und Öffentlichkeitsarbeiten jeder Art sind gebündelt und entwickeln eine bewundernswerte internationale Wirkungskraft.

Zahlreich sind die Schilderungen des Werkes von Paul Klee in seinen vielfältigen Ausrichtungen, wobei oft auf die terminale Erkrankung Bezug genommen wird. Diesmal steht die Krankheit im Mittelpunkt und wird in Bezug zum Alterswerk und zur Biographie gesetzt. Der Autor, Dr. med. Hans Suter, erfuhr seine Ausbildung zum Hautarzt in Bern und Zürich und praktizierte in Thun. Er ist deshalb besonders dazu geeignet. Sorgfäl- tig und behutsam, liebevoll und fast andächtig, allen Spuren und Hinweisen folgend, hat er die medizinischen, die familiären und die künstlerischen Quellen gesichtet, kritisch geprüft und zu einer Gesamtschau der endlich zum Tode führenden Sklerodermie von Paul Klee gefügt. Ein hervorragendes, ein gelungenes Werk! Hervorzuheben sind zwei besondere Anliegen. Zum einen geht der Autor behutsam und sehr eindrücklich auf die Beziehungen ein zwischen dem Befinden des Künstlers, festgehalten in autobiographischen Passagen, und dem künstlerischen Ausdruck, Beziehungen, die sich in vielen Zeichnungen und Bildern der letzten sehr produktiven Jahre finden. Diese erweiterte Sicht auf die letzte Phase im Schaffen von Paul Klee ist durch die Bemühungen von Hans Suter gelungen und weitet unser Verständnis bedeutsam aus. Zum Zweiten gewinnt er aus den vorliegenden Dokumenten die größtmögliche Fülle medizinischer Daten und damit eine gewisse Sicherheit in der Diagnose einer progredient verlaufenden diffusen Sklerodermie. Er vermag damit der mehrfach geäußerten Hypothese einer Mischkollagenose sachlich entgegenzutreten und dieser zu widersprechen.

Das mit reichhaltigen Dokumenten und Bildern geschmückte Buch vermag zu begeistern. Jeder Leser wird dies erfahren. Weite Verbreitung und Berücksichtigung ist ihm zu wünschen, in Kreisen der Kulturschaffenden, der Künstler und der Mediziner.

E. G. Jung, Heidelberg 